\title{
Thermochemical Investigations in the System Cadmium-Praseodymium Relevant for Pyrometallurgical Fuel Reprocessing
}

\author{
THOMAS L. REICHMANN and HERBERT IPSER
}

\begin{abstract}
Vapor pressure measurements, in terms of a (non-)isothermal isopiestic method, were carried out in the system Cd-Pr between $749 \mathrm{~K}$ and $1067 \mathrm{~K}\left(476{ }^{\circ} \mathrm{C}\right.$ and $\left.794{ }^{\circ} \mathrm{C}\right)$. Thermodynamic activities of cadmium as a function of temperature were obtained directly for the composition ranging from 50.0 to 85.7 at. pct $\mathrm{Cd}$. From these results, partial molar enthalpies of mixing of $\mathrm{Cd}$ were derived for the corresponding composition range. The activity values of $\mathrm{Cd}$ were converted to an average sample temperature of $823 \mathrm{~K}\left(550^{\circ} \mathrm{C}\right)$ by applying an integrated form of the Gibbs-Helmholtz equation. These data indicate that $\mathrm{Cd}_{2} \mathrm{Pr}$ and $\mathrm{Cd}_{58} \operatorname{Pr}_{13}$ are probably the most stable intermetallic compounds in this system. Using an activity value of Pr from the literature as integration constant, Gibbs-Duhem integration was performed, and integral Gibbs energies are presented at $823 \mathrm{~K}\left(550{ }^{\circ} \mathrm{C}\right)$, referred to $\mathrm{Cd}(1)$ and $\alpha$ - $\operatorname{Pr}(\mathrm{s})$. Gibbs energies of formation at the stoichiometric compositions of the phases $\mathrm{Cd}_{6} \operatorname{Pr}, \mathrm{Cd}_{58} \mathrm{Pr}_{13}, \mathrm{Cd}_{45} \operatorname{Pr}_{11}, \mathrm{Cd}_{3} \operatorname{Pr}$, and $\mathrm{Cd}_{2} \mathrm{Pr}$ were determined to be about $-18.8,-23.5,-24.8,-28.7$, and $-33.8 \mathrm{~kJ} \mathrm{g-atom}^{-1}$ at $823 \mathrm{~K}\left(550{ }^{\circ} \mathrm{C}\right)$, respectively.
\end{abstract}

DOI: $10.1007 / \mathrm{s} 11661-013-2065-4$

(c) The Minerals, Metals \& Materials Society and ASM International 2013

\section{INTRODUCTION}

NowADAYS several countries in the world strive for strong nuclear power programs to satisfy their demands of energy. As a consequence, according to the World Nuclear Association* around 12,000 tons of

*http://world-nuclear.org/.

high-level nuclear waste is produced annually. Currently, a considerable amount is stored intermediately in on-site water pools, waiting for long-term deposition in geological repositories. For an adequate and economic utilization of nuclear fuels, reprocessing of spent nuclear fuels is an option which is currently adopted only in a few countries. Conventionally, reprocessing is practiced by means of solvent extraction of actinides using tributyl phosphate (TBP), known as hydrometallurgical technique or aqueous reprocessing. Unfortunately, this technique has to deal with several problems like radiation and temperature instability of the various solvents used in the process. In addition, a huge amount of liquid waste is produced by this method. This problem makes it reasonable to investigate another type of reprocessing technique, called the pyrometallurgical technique. Pyrometallurgical reprocessing overcomes some of the major

THOMAS L. REICHMANN, Project Worker, and HERBERT IPSER, Professor, are with the Department of Inorganic Chemistry (Materials Chemistry), University of Vienna, 1090 Wien, Austria. Contact e-mail: Thomas.Reichmann@univie.ac.at

Manuscript submitted May 6, 2013.

Article published online October 18, 2013 problems of aqueous reprocessing, as described by Olander. ${ }^{[1]}$

The central part of pyrometallurgical reprocessing is the electrorefining step, which is carried out in an "electrotransporter" cell. The theoretical feasibility has been described repeatedly in the literature, see e.g., References 2 through 5. In particular, electrotransport and reductive extraction are applied to separate actinides and lanthanides from high level radioactive waste (HLW). The respective electrochemical vessel contains a liquid metal pool at the bottom, covered by a molten salt solution serving as electrolyte. One basket of anode, containing chopped nuclear fuels, and at least two cathodes are inserted into the liquid salt. During this process, especially uranium, plutonium, and minor actinides are transported whereas rare-earth (RE) elements, alkaline, and alkaline earth elements remain in the liquid salt. Additional reductive agents are added to the salt which promotes the extractability of RE elements into the liquid metal pool at the bottom by forming intermetallic compounds. Moriyama et al. ${ }^{[6,7]}$ determined that the separation factors, which are an indicator for extractability, are quite different between actinides and lanthanides. In principle, actinides have the higher affinity for extraction into a metal phase, a fact that is preferable, considering the chemical similarity to lanthanides. The extraction behaviors of different elements between a molten chloride salt phase and a liquid metal strongly depend on the standard free energy of formation of the corresponding chlorides, as well as on the activity coefficients of the extracted metals in the respective intermetallic compounds. Thus, the separation factors differ noticeably when using different liquid metals. Several low melting metals have been tested, 
such as $\mathrm{Al},{ }^{[8]} \mathrm{Bi},{ }^{\left[{ }^{[]}\right.}$and $\mathrm{Cd} .{ }^{[9]}$ Among these elements, $\mathrm{Cd}$ is the most promising metal because it has a rather high vapor pressure, making it convenient for removal by distillation, and it forms a series of rather stable intermetallic compounds with all RE elements. Therefore, a detailed knowledge of the respective Cd-RE phase diagrams as well as of the thermodynamic stabilities of the corresponding intermetallic compounds is of great importance. This was the reason behind our initiating a series of thermodynamic and phase diagram studies of different Cd-RE systems in our laboratory. ${ }^{[10]}$

Concerning the system Cd-Pr, only limited information about phase diagram and thermodynamic data was found in the literature. Although no complete phase diagram information is available, seven intermetallic compounds were reported which seem to have been well investigated. ${ }^{[1]}$ The most Cd-rich intermetallic compound in this system is $\mathrm{Cd}_{11} \mathrm{Pr}$ which decomposes incongruently at $843 \mathrm{~K}\left(570{ }^{\circ} \mathrm{C}\right)$. At this temperature, 3.5 at. pet $\mathrm{Pr}$ is soluble in liquid $\mathrm{Cd}$ according to Johnson et al. ${ }^{[12]}$ The latter authors reported also liquidus data in the composition range of up to 1.83 at. pct $\mathrm{Pr}$, as determined by chemical analysis of filtered samples of the corresponding equilibrium liquid phases.

In a subsequent study, Johnson and Yonco ${ }^{[13]}$ determined the Gibbs energy of formation of $\mathrm{Cd}_{11} \mathrm{Pr}$ by means of Gibbs-Duhem integration, using thermodynamic activity values of $\mathrm{Pr}$ from their own emf measurements. The value, given as $-11.2 \mathrm{~kJ} \mathrm{~g}$-atom $^{-1}$ at $823 \mathrm{~K}\left(550{ }^{\circ} \mathrm{C}\right)$, served as an integration constant for the current calculations. In an early study of Castrillejo et al. ${ }^{[14]}$ Gibbs energies of formation of the three intermetallic compounds $\mathrm{Cd}_{11} \mathrm{Pr}, \mathrm{Cd}_{6} \mathrm{Pr}$, and $\mathrm{Cd}_{58} \operatorname{Pr}_{13}$ were examined by electrochemical techniques. These values were determined to be $-11.2 \pm 0.1,-18.7 \pm 0.1$, and $-22.9 \pm 0.1 \mathrm{~kJ}$ g-atom ${ }^{-1}$, respectively, for a temperature of $823 \mathrm{~K}\left(550^{\circ} \mathrm{C}\right)$. The authors presented also partial Gibbs energies of $\mathrm{Pr}$ in the two-phase fields $\mathrm{Cd}_{58} \operatorname{Pr}_{13}+\mathrm{Cd}_{6} \operatorname{Pr}, \mathrm{Cd}_{6} \operatorname{Pr}+\mathrm{Cd}_{11} \operatorname{Pr}$, and $\mathrm{Cd}_{11} \operatorname{Pr}+\mathrm{L}$, given as $-107.6 \pm 0.6,-127.4 \pm 0.9$, and $-133.8 \pm$ $1.2 \mathrm{~kJ} \mathrm{~mol}(\mathrm{Pr})^{-1}$, respectively, for $823 \mathrm{~K}\left(550{ }^{\circ} \mathrm{C}\right)$.

Due to the limited information from the literature, the aim of the current study was to provide an experimental thermodynamic description of the Cd-Pr system. Vapor pressure measurements were carried out, and thermodynamic activities of $\mathrm{Cd}$ and $\mathrm{Pr}$ as well as integral Gibbs energies were determined for the composition ranging from 50 to 100 at. pct $\mathrm{Cd}$. These data can serve as input into a CALPHAD-type optimization and will subsequently support understanding the distribution behavior of $\mathrm{Pr}$ in the electrorefining cell.

\section{EXPERIMENTAL}

A (non-)isothermal isopiestic method was applied to determine $\mathrm{Cd}$ activities in Cd-Pr alloys. This method was previously described by Ipser et $\mathrm{al}^{[15]}$ The corresponding experimental arrangement is shown schematically in Figure 1. The entire setup is made of fused silica glass and consists of four parts. A silica glass crucible, where

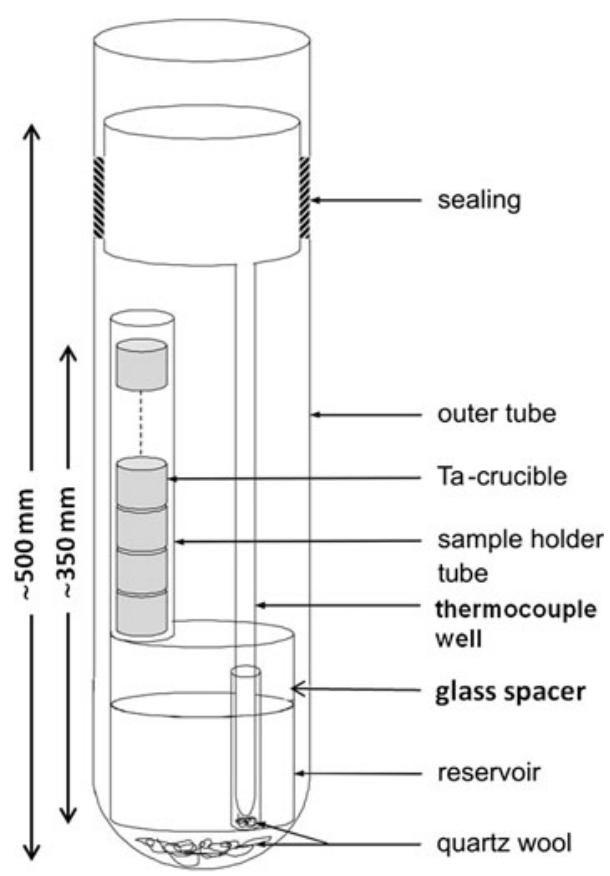

Fig. 1-Isopiestic quartz glass apparatus used in the current study.

the volatile metal is held (the reservoir), is placed at the bottom of the outer tube. On top of this crucible, a glass spacer connected to a sample holder tube is located in which tantalum crucibles are stacked, with one on top of the other. The defined distance from the bottom of the reservoir to the uppermost crucible is around $350 \mathrm{~mm}$. Another inner tube with its upper end widened, is used as a thermocouple well. This widened part is sealed with the outer tube under dynamic vacuum.

Before use, the entire apparatus is cleaned with an acid mixture $\left(\mathrm{HF} / \mathrm{HNO}_{3} / \mathrm{H}_{2} \mathrm{O}\right)$, rinsed with distilled water and dried. Afterward, the completely assembled setup, including the empty Ta crucibles $(-20)$, is degassed under dynamic vacuum $\left(10^{-3}\right.$ mbar $)$ at $1173 \mathrm{~K}\left(900{ }^{\circ} \mathrm{C}\right)$ for 5 hours. The subsequent sample preparation is carried out in a glove box, filled with Ar, to prevent the pure Pr samples from oxidation. Depending on the experimental temperatures, the reservoir is filled with weights ranging between 25 and $35 \mathrm{~g}$ of $\mathrm{Cd}$ (99.9999 pet Alfa AESAR, Karlsruhe, Germany), and between 160 and $220 \mathrm{mg}$ of pure Pr (99.9 pct Alfa AESAR, Karlsruhe, Germany, and smart-elements, Vienna, Austria) is weighed into each Ta crucible (10 mm o.d., $12 \mathrm{~mm}$ height) with an accuracy of \pm 0.1 mg. Thereafter, the assembled setup is securely closed with a glass stopper and shuttled out of the glove box. It is connected to a vacuum pump, evacuated, and sealed under a dynamic vacuum of better than $10^{-4}$ mbar.

Afterward, the isopiestic experiments are heated in different temperature gradients, as applicable in conventional two-zone furnaces. A total number of seven runs were performed with different reservoir $\left(T_{\mathrm{R}}\right)$ and sample temperatures $\left(T_{\mathrm{S}}\right)$. The respective temperature gradients were recorded by raising a $\mathrm{Pt} / \mathrm{Pt} 10$ pet $\mathrm{Rh}$ thermocouple inside the thermocouple well. Each experiment lasted for about 3 to 5 weeks, depending on the 
reservoir temperature. After equilibration, the isopiestic apparatus was quenched in cold water and cut open by a diamond saw. The Cd-Pr alloys which had formed within the Ta crucibles during equilibration with the $\mathrm{Cd}$ vapor were weighed back and their compositions calculated from the difference in weight, which was attributed to the uptake of $\mathrm{Cd}$.

Representative samples were characterized by powder $\mathrm{X}$-ray diffraction (XRD) with $\mathrm{Cu}-\mathrm{K} \alpha$ radiation using a Bruker D8 Advance Diffractometer with Bragg-Brentano geometry. The corresponding XRD patterns were analyzed and refined by means of the TOPAS 3 software, applying the fundamental parameter approach for peak profile modeling. To protect the powdered alloys from oxidation, a special XRD sample holder with an X-ray transparent lid was used.

Quantitative and optical examinations of the microstructures were performed on a Zeiss Supra 55 VP environmental scanning electron microscope using pure elements as standard materials and Co for an energy calibration of the energy-dispersive X-ray detector signal. A $120 \mu \mathrm{m}$ aperture was used, and an acceleration voltage of $20 \mathrm{kV}$ was applied. For imaging of the microstructures, a backscatter detector was employed. The composition of each phase was measured at three or more spots to minimize statistical errors and to obtain more reliable results. A maximum error of about \pm 0.5 at. pct is assumed for the final results.

\section{RESULTS AND DISCUSSION}

\section{A. (Non-) isothermal Isopiestic Measurements}

Seven isopiestic runs in total were carried out at different temperature conditions, to obtain a complete description of thermodynamic activity data in the Cd-Pr system. The corresponding reservoir temperatures were chosen between $729 \mathrm{~K}$ and $869 \mathrm{~K}\left(456{ }^{\circ} \mathrm{C}\right.$ and $\left.596{ }^{\circ} \mathrm{C}\right)$, with the corresponding $\mathrm{Cd}$ vapor pressures being between 7 and 101 mbar, respectively. Sample temperatures were located between $749 \mathrm{~K}$ and $1067 \mathrm{~K}\left(476^{\circ} \mathrm{C}\right.$ and $794^{\circ} \mathrm{C}$ ). The vapor pressure values of pure $\mathrm{Cd}$ as a function of temperature were taken from Binnewies and Milke $^{[16]}$ :

$$
\log \left(\frac{p_{\mathrm{Cd}}^{0}}{\mathrm{bar}}\right)=8.7-5690 \cdot \frac{\mathrm{K}}{T}-1.07 \cdot \log \frac{T}{\mathrm{~K}}
$$

Since the melting point of $\mathrm{Cd}$ is rather low and it has indeed a much higher vapor pressure than Pr at the respective reservoir temperatures, the total pressure in the system is determined only by the temperature of the $\mathrm{Cd}$ reservoir $\left(T_{\mathrm{R}}\right)$. Equilibrium within the system is reached when the $\mathrm{Cd}$ vapor pressure over each sample, at its sample temperature $T_{\mathrm{S}}$, is equal to the vapor pressure of pure $\mathrm{Cd}$ at the reservoir temperature $T_{\mathrm{R}}$ :

$$
p_{\mathrm{Cd}}\left(T_{\mathrm{S}}\right)=p_{\mathrm{Cd}}^{0}\left(T_{\mathrm{R}}\right)
$$

Thus, the thermodynamic activity of $\mathrm{Cd}$ for each sample can be obtained by combining Eqs. [1] and [2] based on the conventional definition for the activity:

$$
a_{\mathrm{Cd}}\left(T_{\mathrm{S}}\right)=\frac{p_{\mathrm{Cd}}\left(T_{\mathrm{S}}\right)}{p_{\mathrm{Cd}}^{0}\left(T_{\mathrm{S}}\right)}=\frac{p_{\mathrm{Cd}}^{0}\left(T_{\mathrm{R}}\right)}{p_{\mathrm{Cd}}^{0}\left(T_{\mathrm{S}}\right)}
$$

All results, including sample temperatures and compositions, sample identifications, $\mathrm{Cd}$ activities, and partial enthalpy values, are listed in Table I. The compositions were calculated from the mass change during the experiment as described above. All samples were ascribed to one- or two-phase fields in the phase diagram, which was confirmed by powder XRD. Sample compositions were partially controlled by SEM, and both sets of results were found to be in good agreement. The relative uncertainty in the compositions due to experimental errors is estimated to be less than 0.5 at. pct, and the temperatures are assumed to be accurate within $\pm 2 \mathrm{~K}$ (A more detailed discussion on possible error sources in this type of isopiestic experiments can be found in Reference 15).

Although the results were consistent in general, some discrepancies were found, predominantly for isopiestic runs with higher reservoir temperatures. Accordingly, the homogeneity ranges of all phases in the Cd-Pr system were separately determined by SEM. For this purpose, isopiestic samples within two-phase fields were annealed at different temperatures. The resulting solubilities for $823 \mathrm{~K}\left(550{ }^{\circ} \mathrm{C}\right)$ are shown in Table II. Although $\mathrm{Cd}_{11} \mathrm{Pr}$ and $\mathrm{Cd}_{6} \mathrm{Pr}$ were exclusively determined as line compounds, noticeable homogeneity ranges were found for the other compounds. All isopiestic samples, except those from run 7 , covered the experimentally found homogeneity ranges of the different phases. Most of the calculated compositions of run 7 appeared, in contrast to the other runs, to be located in two-phase fields as well as systematically shifted toward Pr-rich compositions. The corresponding SEM investigations suggested that, during quenching from $869 \mathrm{~K}\left(596^{\circ} \mathrm{C}\right)$, some $\mathrm{Cd}$ was lost from the surface of the particles in all the samples of run 7, as can be seen in the example of sample 11 in Figure 2(a). This loss is caused by the rather significant $\mathrm{Cd}$ partial pressure at this high reservoir temperature and by the finite quenching rate of the evacuated isopiestic tubes. Although the overall composition could not be measured reliably because of porosity, powder XRD pointed to $\mathrm{Cd}_{58} \operatorname{Pr}_{13}$ as the bulk phase ( $c f$. Figure 3 ). Therefore, it is strongly assumed that sample 11 was located within the homogeneity range of $\mathrm{Cd}_{58} \operatorname{Pr}_{13}$ while being equilibrated and was shifted in composition only during quenching.

Another possible explanation as to why these calculated compositions were shifted could be that a continuous solid solution exists between the two phases $\mathrm{Cd}_{58} \operatorname{Pr}_{13}$ and $\mathrm{Cd}_{45} \operatorname{Pr}_{11}$ at elevated temperatures, especially at $823 \mathrm{~K}\left(550{ }^{\circ} \mathrm{C}\right)$. However, due to pronounced differences in the crystal structures and because both phases were found in thermodynamic equilibrium at $873 \mathrm{~K}\left(600^{\circ} \mathrm{C}\right)$, as seen in Figure $2(\mathrm{~b})$, such a continuous solid solubility is very unlikely. Therefore, all sample compositions of run 7 were shifted systematically 0.35 at. pet toward the Cd-rich side.

By plotting sample compositions against sample temperatures $T_{\mathrm{S}}$ for one particular run, the so-called equilibrium curve can be drawn connecting the individ- 
Table I. Experimental Results of Isopiestic Experiments, Standard State: Cd(I)

\begin{tabular}{|c|c|c|c|c|c|c|}
\hline Sample No. & Cd (at. pct) & $T_{\mathrm{S}} / \mathrm{K}$ & $\ln a_{\mathrm{Cd}}\left(T_{\mathrm{S}}\right)$ & Phases & $\Delta \bar{H}_{\mathrm{Cd}} / \mathrm{kJ}$ g-atom $^{-1}$ & $\ln a_{\mathrm{Cd}}\left[823 \mathrm{~K}\left(550^{\circ} \mathrm{C}\right)\right]$ \\
\hline \multicolumn{7}{|c|}{ Run 1: $T_{\mathrm{R}}=841 \mathrm{~K}\left(568^{\circ} \mathrm{C}\right), 41$ days } \\
\hline 1 & 84.88 & 850 & -0.16 & $\mathrm{Cd}_{58} \operatorname{Pr}_{13}+\mathrm{Cd}_{6} \mathrm{Pr}$ & -20.5 & -0.29 \\
\hline 2 & 81.49 & 856 & -0.25 & $\mathrm{Cd}_{58} \operatorname{Pr}_{13}$ & -23.9 & -0.39 \\
\hline 3 & 81.24 & 864 & -0.39 & $\mathrm{Cd}_{58} \operatorname{Pr}_{13}$ & -28.7 & -0.59 \\
\hline 4 & 80.94 & 875 & -0.56 & $\mathrm{Cd}_{58} \operatorname{Pr}_{13}$ & -40.9 & -0.92 \\
\hline 5 & 80.83 & 890 & -0.79 & $\mathrm{Cd}_{58} \operatorname{Pr}_{13}$ & -45.5 & -1.29 \\
\hline 6 & 79.89 & 908 & -1.06 & $\mathrm{Cd}_{3} \operatorname{Pr}+\mathrm{Cd}_{45} \operatorname{Pr}_{11}$ & -30.2 & -1.48 \\
\hline 7 & 73.77 & 926 & -1.33 & $\mathrm{Cd}_{2} \mathrm{Pr}+\mathrm{Cd}_{3} \mathrm{Pr}$ & -39.3 & -1.97 \\
\hline 8 & 66.61 & 950 & -1.66 & $\mathrm{Cd}_{2} \mathrm{Pr}$ & -8.70 & -1.83 \\
\hline 9 & 66.47 & 969 & -1.91 & $\mathrm{Cd}_{2} \mathrm{Pr}$ & -1.80 & -1.95 \\
\hline 10 & 66.43 & 992 & -2.19 & $\mathrm{Cd}_{2} \mathrm{Pr}$ & -0.55 & -2.21 \\
\hline 11 & 65.59 & 1012 & -2.44 & $\mathrm{CdPr}+\mathrm{Cd}_{2} \mathrm{Pr}$ & -66.0 & -4.24 \\
\hline \multicolumn{7}{|c|}{ Run 2: $T_{\mathrm{R}}=818 \mathrm{~K}\left(545^{\circ} \mathrm{C}\right), 49$ days } \\
\hline 1 & 79.44 & 872 & -0.92 & $\mathrm{Cd}_{3} \operatorname{Pr}+\mathrm{Cd}_{45} \operatorname{Pr}_{11}$ & -30.2 & -1.17 \\
\hline 2 & 73.89 & 885 & -1.12 & $\mathrm{Cd}_{2} \mathrm{Pr}+\mathrm{Cd}_{3} \mathrm{Pr}$ & -39.3 & -1.53 \\
\hline 3 & 66.79 & 899 & -1.34 & $\mathrm{Cd}_{2} \mathrm{Pr}+\mathrm{Cd}_{3} \mathrm{Pr}$ & -39.3 & -1.87 \\
\hline 4 & 66.64 & 913 & -1.55 & $\mathrm{Cd}_{2} \mathrm{Pr}$ & -10.2 & -1.70 \\
\hline 5 & 66.50 & 929 & -1.78 & $\mathrm{Cd}_{2} \mathrm{Pr}$ & -3.33 & -1.84 \\
\hline 6 & 66.48 & 942 & -1.96 & $\mathrm{Cd}_{2} \mathrm{Pr}$ & -2.29 & -2.00 \\
\hline 7 & 66.41 & 957 & -2.16 & $\mathrm{Cd}_{2} \mathrm{Pr}$ & 0.12 & -2.16 \\
\hline 8 & 66.19 & 974 & -2.38 & $\mathrm{Cd}_{2} \mathrm{Pr}$ & 3.51 & -2.30 \\
\hline 9 & 65.43 & 995 & -2.64 & $\mathrm{CdPr}+\mathrm{Cd}_{2} \mathrm{Pr}$ & -66.0 & -4.31 \\
\hline 10 & 57.62 & 1018 & -2.91 & $\mathrm{CdPr}+\mathrm{Cd}_{2} \mathrm{Pr}$ & -66.0 & -4.74 \\
\hline \multicolumn{7}{|c|}{ Run 3: $T_{\mathrm{R}}=802 \mathrm{~K}\left(529^{\circ} \mathrm{C}\right), 35$ days } \\
\hline 1 & 81.22 & 825 & -0.43 & $\mathrm{Cd}_{58} \operatorname{Pr}_{13}$ & -29.5 & -0.44 \\
\hline 2 & 81.10 & 831 & -0.54 & $\mathrm{Cd}_{58} \operatorname{Pr}_{13}$ & -34.2 & -0.59 \\
\hline 3 & 80.98 & 837 & -0.64 & $\mathrm{Cd}_{58} \operatorname{Pr}_{13}$ & -39.4 & -0.74 \\
\hline 4 & 80.78 & 843 & -0.75 & $\mathrm{Cd}_{58} \operatorname{Pr}_{13}$ & -47.6 & -0.92 \\
\hline 5 & 80.73 & 850 & -0.86 & $\mathrm{Cd}_{58} \operatorname{Pr}_{13}$ & -49.0 & -1.08 \\
\hline 6 & 80.70 & 858 & -0.99 & $\mathrm{Cd}_{58} \operatorname{Pr}_{13}$ & -49.9 & -1.28 \\
\hline 7 & 80.53 & 865 & -1.10 & $\mathrm{Cd}_{45} \operatorname{Pr}_{11}+\mathrm{Cd}_{58} \operatorname{Pr}_{13}$ & -38.1 & -1.38 \\
\hline 8 & 79.61 & 872 & -1.22 & $\mathrm{Cd}_{3} \mathrm{Pr}+\mathrm{Cd}_{45} \operatorname{Pr}_{11}$ & -30.2 & -1.47 \\
\hline 9 & 77.02 & 881 & -1.36 & $\mathrm{Cd}_{3} \operatorname{Pr}+\mathrm{Cd}_{45} \operatorname{Pr}_{11}$ & -30.2 & -1.66 \\
\hline 10 & 69.47 & 892 & -1.53 & $\mathrm{Cd}_{2} \mathrm{Pr}+\mathrm{Cd}_{3} \mathrm{Pr}$ & -39.3 & -1.96 \\
\hline 11 & 66.54 & 906 & -1.74 & $\mathrm{Cd}_{2} \mathrm{Pr}$ & -4.78 & -1.53 \\
\hline 12 & 66.35 & 920 & -1.94 & $\mathrm{Cd}_{2} \mathrm{Pr}$ & 1.70 & -2.01 \\
\hline 13 & 66.32 & 937 & -2.19 & $\mathrm{Cd}_{2} \mathrm{Pr}$ & 2.23 & -2.26 \\
\hline 14 & 66.30 & 855 & -2.43 & $\mathrm{Cd}_{2} \mathrm{Pr}$ & 2.56 & -2.51 \\
\hline 15 & 66.18 & 973 & -2.66 & $\mathrm{Cd}_{2} \mathrm{Pr}$ & 3.52 & -2.76 \\
\hline 16 & 66.01 & 990 & -2.88 & $\mathrm{Cd}_{2} \mathrm{Pr}$ & 1.54 & -2.91 \\
\hline 17 & 65.82 & 1005 & -3.06 & $\mathrm{CdPr}+\mathrm{Cd}_{2} \mathrm{Pr}$ & -66.0 & -4.81 \\
\hline 18 & 60.72 & 1017 & -3.20 & $\mathrm{CdPr}+\mathrm{Cd}_{2} \mathrm{Pr}$ & -66.0 & -5.04 \\
\hline \multicolumn{7}{|c|}{ Run 4: $T_{\mathrm{R}}=780 \mathrm{~K}\left(507^{\circ} \mathrm{C}\right), 42$ days } \\
\hline 1 & 84.54 & 800 & -0.40 & $\mathrm{Cd}_{58} \operatorname{Pr}_{13}+\mathrm{Cd}_{6} \mathrm{Pr}$ & -20.5 & -0.35 \\
\hline 2 & 83.55 & 804 & -0.47 & $\mathrm{Cd}_{58} \operatorname{Pr}_{13}+\mathrm{Cd}_{6} \mathrm{Pr}$ & -20.5 & -0.35 \\
\hline 3 & 81.27 & 808 & -0.54 & $\mathrm{Cd}_{58} \operatorname{Pr}_{13}$ & -27.9 & -0.47 \\
\hline 4 & 81.16 & 811 & -0.61 & $\mathrm{Cd}_{58} \operatorname{Pr}_{13}$ & -31.8 & -0.54 \\
\hline 5 & 81.09 & 814 & -0.66 & $\mathrm{Cd}_{58} \operatorname{Pr}_{13}$ & -34.3 & -0.60 \\
\hline 6 & 81.03 & 817 & -0.72 & $\mathrm{Cd}_{58} \operatorname{Pr}_{13}$ & -37.1 & -0.68 \\
\hline 7 & 81.06 & 820 & -0.76 & $\mathrm{Cd}_{58} \operatorname{Pr}_{13}$ & -35.9 & -0.75 \\
\hline 8 & 80.98 & 822 & -0.81 & $\mathrm{Cd}_{58} \operatorname{Pr}_{13}$ & -39.5 & -0.80 \\
\hline 9 & 80.92 & 825 & -0.86 & $\mathrm{Cd}_{58} \operatorname{Pr}_{13}$ & -41.9 & -0.87 \\
\hline 10 & 80.92 & 828 & -0.91 & $\mathrm{Cd}_{58} \operatorname{Pr}_{13}$ & -41.9 & -0.94 \\
\hline 11 & 81.00 & 832 & -0.98 & $\mathrm{Cd}_{58} \operatorname{Pr}_{13}$ & -38.5 & -1.04 \\
\hline 12 & 80.97 & 837 & -1.07 & $\mathrm{Cd}_{58} \operatorname{Pr}_{13}$ & -39.9 & -1.17 \\
\hline 13 & 80.41 & 847 & -1.24 & $\mathrm{Cd}_{45} \operatorname{Pr}_{11}+\mathrm{Cd}_{58} \operatorname{Pr}_{13}$ & -38.1 & -1.40 \\
\hline 14 & 78.86 & 858 & -1.42 & $\mathrm{Cd}_{3} \operatorname{Pr}+\mathrm{Cd}_{45} \operatorname{Pr}_{11}$ & -30.2 & -1.58 \\
\hline 15 & 69.47 & 871 & -1.63 & $\mathrm{Cd}_{2} \mathrm{Pr}+\mathrm{Cd}_{3} \mathrm{Pr}$ & -39.3 & -1.95 \\
\hline 16 & 66.57 & 882 & -1.82 & $\mathrm{Cd}_{2} \mathrm{Pr}$ & -6.56 & -1.88 \\
\hline 17 & 66.48 & 894 & -1.99 & $\mathrm{Cd}_{2} \mathrm{Pr}$ & -2.46 & -2.02 \\
\hline 18 & 66.43 & 904 & -2.14 & $\mathrm{Cd}_{2} \mathrm{Pr}$ & -0.60 & -2.15 \\
\hline 19 & 66.42 & 914 & -2.30 & $\mathrm{Cd}_{2} \mathrm{Pr}$ & -0.35 & -2.30 \\
\hline 20 & 66.41 & 922 & -2.41 & $\mathrm{Cd}_{2} \mathrm{Pr}$ & 0.18 & -2.41 \\
\hline 21 & 66.30 & 930 & -2.52 & $\mathrm{Cd}_{2} \mathrm{Pr}$ & 2.64 & -2.48 \\
\hline
\end{tabular}


Table I. continued

\begin{tabular}{|c|c|c|c|c|c|c|}
\hline Sample No. & Cd (at. pct) & $T_{\mathrm{S}} / \mathrm{K}$ & $\ln a_{\mathrm{Cd}}\left(T_{\mathrm{S}}\right)$ & Phases & $\Delta \bar{H}_{\mathrm{Cd}} / \mathrm{kJ}$ g-atom $^{-1}$ & $\ln a_{\mathrm{Cd}}\left[823 \mathrm{~K}\left(550^{\circ} \mathrm{C}\right)\right]$ \\
\hline \multicolumn{7}{|c|}{ Run 5: $T_{\mathrm{R}}=795 \mathrm{~K}\left(522^{\circ} \mathrm{C}\right), 45$ days } \\
\hline 1 & 81.22 & 808 & -0.25 & $\mathrm{Cd}_{58} \operatorname{Pr}_{13}$ & -29.4 & -0.18 \\
\hline 2 & 81.02 & 818 & -0.44 & $\mathrm{Cd}_{58} \operatorname{Pr}_{13}$ & -37.6 & -0.41 \\
\hline 3 & 80.89 & 827 & -0.60 & $\mathrm{Cd}_{58} \operatorname{Pr}_{13}$ & -43.1 & -0.63 \\
\hline 4 & 80.74 & 837 & -0.78 & $\mathrm{Cd}_{58} \operatorname{Pr}_{13}$ & -48.6 & -1.90 \\
\hline 5 & 80.66 & 845 & -0.91 & $\mathrm{Cd}_{58} \operatorname{Pr}_{13}$ & -51.0 & $-1.11 *$ \\
\hline 6 & 80.56 & 854 & -1.06 & $\mathrm{Cd}_{58} \operatorname{Pr}_{13}$ & -52.7 & $-1.33^{*}$ \\
\hline 7 & 80.54 & 861 & -1.17 & $\mathrm{Cd}_{45} \operatorname{Pr}_{11}+\mathrm{Cd}_{58} \operatorname{Pr}_{13}$ & -38.1 & -1.42 \\
\hline 8 & 79.53 & 867 & -1.27 & $\mathrm{Cd}_{3} \operatorname{Pr}+\mathrm{Cd}_{45} \operatorname{Pr}_{11}$ & -30.2 & -1.50 \\
\hline 9 & 73.16 & 873 & -1.38 & $\mathrm{Cd}_{2} \mathrm{Pr}+\mathrm{Cd}_{3} \mathrm{Pr}$ & -39.3 & -1.70 \\
\hline 10 & 72.77 & 880 & -1.48 & $\mathrm{Cd}_{2} \mathrm{Pr}+\mathrm{Cd}_{3} \mathrm{Pr}$ & -39.3 & -1.86 \\
\hline 11 & 66.54 & 884 & -1.55 & $\mathrm{Cd}_{2} \mathrm{Pr}$ & -5.08 & -1.61 \\
\hline 12 & 66.63 & 889 & -1.63 & $\mathrm{Cd}_{2} \mathrm{Pr}$ & -9.59 & -1.73 \\
\hline 13 & 66.69 & 894 & -1.69 & $\mathrm{Cd}_{2} \mathrm{Pr}$ & -13.2 & -1.85 \\
\hline \multicolumn{7}{|c|}{ Run 6: $T_{\mathrm{R}}=729 \mathrm{~K}\left(456^{\circ} \mathrm{C}\right), 49$ days } \\
\hline 1 & 85.53 & 749 & -0.45 & $\mathrm{Cd}_{58} \operatorname{Pr}_{13}+\mathrm{Cd}_{6} \operatorname{Pr}$ & -20.5 & -0.16 \\
\hline 2 & 85.30 & 753 & -0.55 & $\mathrm{Cd}_{58} \operatorname{Pr}_{13}+\mathrm{Cd}_{6} \operatorname{Pr}$ & -20.5 & -0.26 \\
\hline 3 & 82.11 & 756 & -0.61 & $\mathrm{Cd}_{58} \operatorname{Pr}_{13}+\mathrm{Cd}_{6} \mathrm{Pr}$ & -20.5 & -0.34 \\
\hline 4 & 81.59 & 759 & -0.67 & $\mathrm{Cd}_{58} \operatorname{Pr}_{13}$ & -24.8 & -0.37 \\
\hline 5 & 81.48 & 761 & -0.72 & $\mathrm{Cd}_{58} \operatorname{Pr}_{13}$ & -24.0 & -0.44 \\
\hline 6 & 81.40 & 763 & -0.76 & $\mathrm{Cd}_{58} \operatorname{Pr}_{13}$ & -24.8 & -0.48 \\
\hline 7 & 81.34 & 765 & -0.80 & $\mathrm{Cd}_{58} \operatorname{Pr}_{13}$ & -25.9 & -0.51 \\
\hline 8 & 81.29 & 766 & -0.82 & $\mathrm{Cd}_{58} \operatorname{Pr}_{13}$ & -27.3 & -0.53 \\
\hline 9 & 81.29 & 767 & -0.84 & $\mathrm{Cd}_{58} \operatorname{Pr}_{13}$ & -27.0 & -0.55 \\
\hline 10 & 81.24 & 768 & -0.86 & $\mathrm{Cd}_{58} \operatorname{Pr}_{13}$ & -28.8 & -0.56 \\
\hline 11 & 81.15 & 771 & -0.91 & $\mathrm{Cd}_{58} \operatorname{Pr}_{13}$ & -32.0 & -0.59 \\
\hline 12 & 81.15 & 775 & -0.99 & $\mathrm{Cd}_{58} \operatorname{Pr}_{13}$ & -31.9 & -0.70 \\
\hline 13 & 81.04 & 781 & -1.13 & $\mathrm{Cd}_{58} \operatorname{Pr}_{13}$ & 36.8 & -0.84 \\
\hline 14 & 80.97 & 791 & -1.32 & $\mathrm{Cd}_{58} \operatorname{Pr}_{13}$ & -39.8 & -1.08 \\
\hline 15 & 80.88 & 801 & -1.52 & $\mathrm{Cd}_{58} \operatorname{Pr}_{13}$ & -43.7 & -1.34 \\
\hline 16 & 78.79 & 810 & -1.68 & $\mathrm{Cd}_{3} \operatorname{Pr}+\mathrm{Cd}_{45} \operatorname{Pr}_{11}$ & -30.2 & -1.59 \\
\hline 17 & 66.53 & 828 & -2.00 & $\mathrm{Cd}_{2} \mathrm{Pr}+\mathrm{Cd}_{3} \mathrm{Pr}$ & -39.3 & -2.02 \\
\hline 18 & 65.96 & 834 & -2.12 & $\mathrm{Cd}_{2} \mathrm{Pr}$ & 0.29 & -2.12 \\
\hline 19 & 65.76 & 842 & -2.25 & $\mathrm{Cd}_{2} \mathrm{Pr}$ & -7.97 & -2.28 \\
\hline \multicolumn{7}{|c|}{ Run 7: $T_{\mathrm{R}}=869 \mathrm{~K}\left(596^{\circ} \mathrm{C}\right), 42$ days } \\
\hline 1 & 81.32 & 888 & -0.30 & $\mathrm{Cd}_{58} \operatorname{Pr}_{13}$ & -26.3 & -0.58 \\
\hline 2 & 81.09 & 893 & -0.38 & $\mathrm{Cd}_{58} \operatorname{Pr}_{13}$ & -34.3 & -0.77 \\
\hline 3 & 81.00 & 897 & -0.44 & $\mathrm{Cd}_{58} \operatorname{Pr}_{13}$ & -38.5 & -0.90 \\
\hline 4 & 80.90 & 902 & -0.51 & $\mathrm{Cd}_{58} \operatorname{Pr}_{13}$ & -42.9 & -1.05 \\
\hline 5 & 80.88 & 906 & -0.58 & $\mathrm{Cd}_{58} \operatorname{Pr}_{13}$ & -43.5 & -1.16 \\
\hline 6 & 80.72 & 911 & -0.65 & $\mathrm{Cd}_{58} \operatorname{Pr}_{13}$ & -49.4 & -1.35 \\
\hline 7 & 80.58 & 916 & -0.72 & $\mathrm{Cd}_{58} \operatorname{Pr}_{13}$ & -52.3 & -1.50 \\
\hline 8 & 80.55 & 922 & -0.80 & $\mathrm{Cd}_{45} \operatorname{Pr}_{11}+\mathrm{Cd}_{58} \operatorname{Pr}_{13}$ & -38.1 & -1.40 \\
\hline 9 & 80.34 & 927 & -0.87 & $\mathrm{Cd}_{45} \operatorname{Pr}_{11}$ & -36.7 & -1.47 \\
\hline 10 & 80.26 & 933 & -0.96 & $\mathrm{Cd}_{45} \operatorname{Pr}_{11}$ & -36.1 & -1.58 \\
\hline 11 & 79.43 & 942 & -1.08 & $\mathrm{Cd}_{3} \operatorname{Pr}+\mathrm{Cd}_{45} \operatorname{Pr}_{11}$ & -30.2 & -1.64 \\
\hline 12 & 75.05 & 954 & -1.24 & $\mathrm{Cd}_{2} \mathrm{Pr}+\mathrm{Cd}_{3} \operatorname{Pr}$ & -39.3 & -2.03 \\
\hline 13 & 66.28 & 967 & -1.42 & $\mathrm{Cd}_{2} \mathrm{Pr}$ & 2.89 & -1.36 \\
\hline 14 & 66.27 & 984 & -1.63 & $\mathrm{Cd}_{2} \mathrm{Pr}$ & 3.01 & -1.56 \\
\hline 15 & 66.07 & 1001 & -1.83 & $\mathrm{Cd}_{2} \mathrm{Pr}$ & 2.72 & -1.76 \\
\hline 16 & 66.22 & 1015 & -2.01 & $\mathrm{Cd}_{2} \mathrm{Pr}$ & 3.43 & -1.91 \\
\hline 17 & 66.17 & 1028 & -2.16 & $\mathrm{Cd}_{2} \mathrm{Pr}$ & 3.52 & -2.05 \\
\hline 18 & 66.02 & 1039 & -2.27 & $\mathrm{Cd}_{2} \mathrm{Pr}$ & 1.83 & -2.22 \\
\hline 19 & 65.75 & 1050 & -2.40 & $\mathrm{Cd}_{2} \mathrm{Pr}$ & -8.62 & -2.67 \\
\hline 20 & 65.78 & 1059 & -2.50 & $\mathrm{Cd}_{2} \mathrm{Pr}$ & -6.99 & -2.73 \\
\hline 21 & 65.84 & 1067 & -2.58 & $\mathrm{CdPr}+\mathrm{Cd}_{2} \mathrm{Pr}$ & -66.0 & -4.78 \\
\hline
\end{tabular}

*Samples are not located within the currently defined homogeneity range of $\mathrm{Cd}_{58} \operatorname{Pr}_{13}$ but were treated as single-phase samples of $\mathrm{Cd}_{58} \operatorname{Pr}_{13}$, confirmed by powder-XRD (compare 3.1.).

ual data points. Figure 4 shows these equilibrium curves for all runs, superimposed on the actual version of the $\mathrm{Cd}-\mathrm{Pr}$ phase diagram, adapted from Gschneidner and
Calderwood. ${ }^{[17]}$ As can be seen, all samples are located between 57 and 86 at. pct $\mathrm{Cd}$. Most of the samples are single-phase samples, consisting of pure $\mathrm{Cd}_{2} \mathrm{Pr}$ and 
$\mathrm{Cd}_{58} \operatorname{Pr}_{13}$, suggesting that these phases are among the most stable ones in the Cd-Pr system. On the other hand, no samples were obtained in the phase $\mathrm{Cd}_{3} \mathrm{Pr}$ suggesting that $\mathrm{Cd}_{3} \mathrm{Pr}$ is only slightly more stable than a two-phase mixture of its neighboring compounds. This phenomenon was actually also observed earlier in the $\mathrm{Cd}-\mathrm{Ce}$ system where likewise no samples were formed in the $\mathrm{Cd}_{3} \mathrm{Ce}$ phase. ${ }^{[10]}$

Table II. Homogeneity Ranges of the Intermetallic Compounds at $823 \mathrm{~K}\left(550^{\circ} \mathrm{C}\right)$ as Determined by SEM

\begin{tabular}{lcc}
\hline Phase & Phase Boundaries (at. pct Cd) & Remarks \\
\hline $\mathrm{CdPr}$ & 47.07 to 50.00 & \\
$\mathrm{Cd}_{2} \mathrm{Pr}$ & 65.80 to 66.67 & \\
$\mathrm{Cd}_{3} \mathrm{Pr}$ & 75.00 to 76.19 & \\
$\mathrm{Cd}_{45} \operatorname{Pr}_{11}$ & 79.98 to 80.36 & \\
$\mathrm{Cd}_{58} \mathrm{Pr}_{13}$ & 80.76 to 81.79 & line compound \\
$\mathrm{Cd}_{6} \mathrm{Pr}$ & 85.71 & line compound \\
$\mathrm{Cd}_{11} \mathrm{Pr}$ & 91.67 & \\
\hline
\end{tabular}

In addition, some of the samples formed in the isopiestic runs were obtained in various two-phase fields after equilibration. This is probably caused by slight variations of the sample temperatures. Nevertheless, these samples allow us to calculate $\mathrm{Cd}$ activities for the respective two-phase fields at the corresponding sample temperatures $T_{\mathrm{S}}$.

\section{B. Partial Enthalpy of Mixing of Cd in Two-Phase Fields}

As indicated above, Cd vapor pressures were used to calculate activities in different two-phase fields at the respective sample temperatures $T_{\mathrm{S}}$ (see Table I). Natural logarithms of these activity values were plotted against reciprocal temperatures for almost all two-phase fields, i.e.,: $\mathrm{CdPr}+\mathrm{Cd}_{2} \mathrm{Pr}, \mathrm{Cd}_{2} \mathrm{Pr}+\mathrm{Cd}_{3} \operatorname{Pr}, \mathrm{Cd}_{3} \mathrm{Pr}+\mathrm{Cd}_{45} \operatorname{Pr}_{11}$, $\mathrm{Cd}_{45} \operatorname{Pr}_{11}+\mathrm{Cd}_{58} \operatorname{Pr}_{13}$, and $\mathrm{Cd}_{58} \operatorname{Pr}_{13}+\mathrm{Cd}_{6} \operatorname{Pr}$ (see Figure 5). According to an adapted Gibbs-Helmholtz equation [4], partial enthalpy values of mixing of $\mathrm{Cd}$ were directly calculated from the slopes:
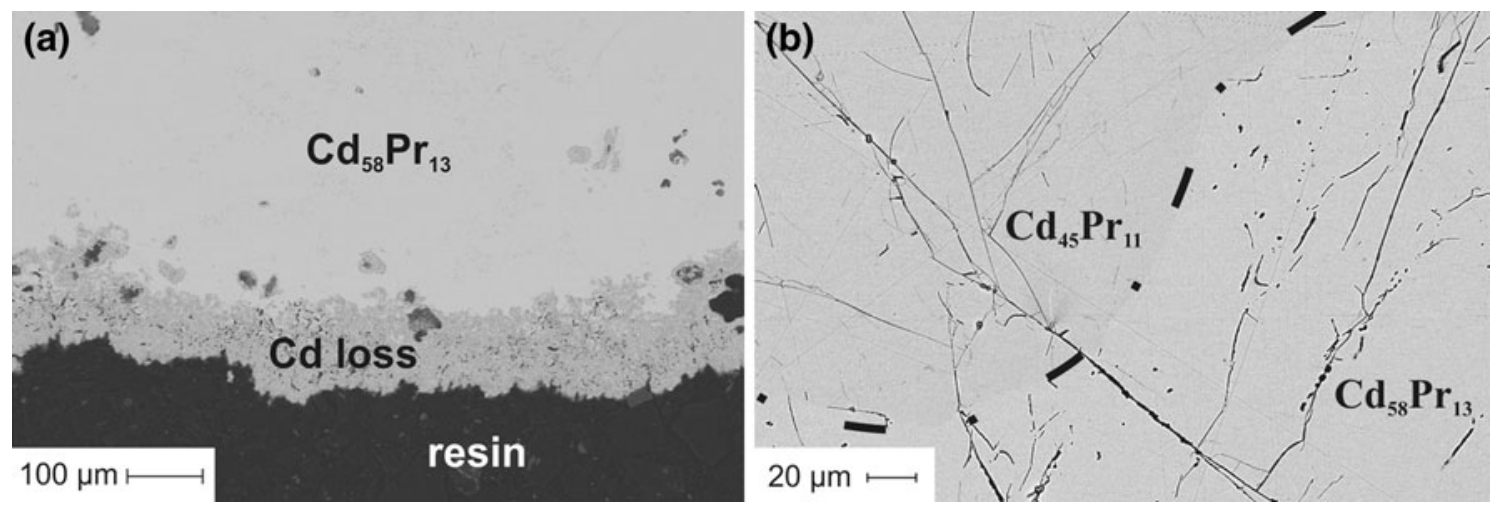

Fig. 2-BSE image of sample 11 from isopiestic run 7 (a) and of a sample annealed in the two-phase field $\mathrm{Cd}_{58} \operatorname{Pr}_{13}+\mathrm{Cd}_{45} \operatorname{Pr}_{11}$ at $873 \mathrm{~K}$ $\left(600{ }^{\circ} \mathrm{C}\right)(b)$.

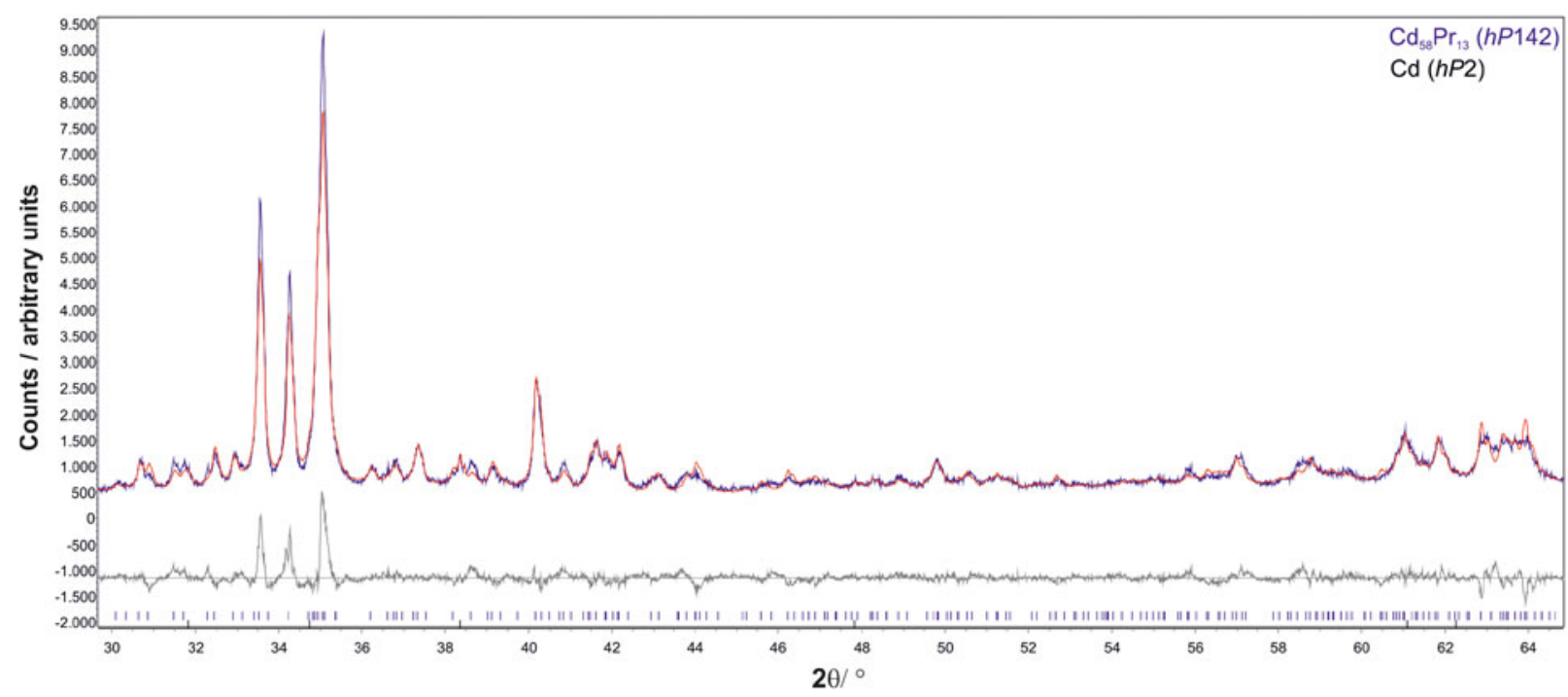

Fig. 3-Powder XRD pattern of sample 11 from isopiestic run 7. Blue curve: observed pattern; red curve: calculated pattern; gray curve: difference pattern (Color figure online). 


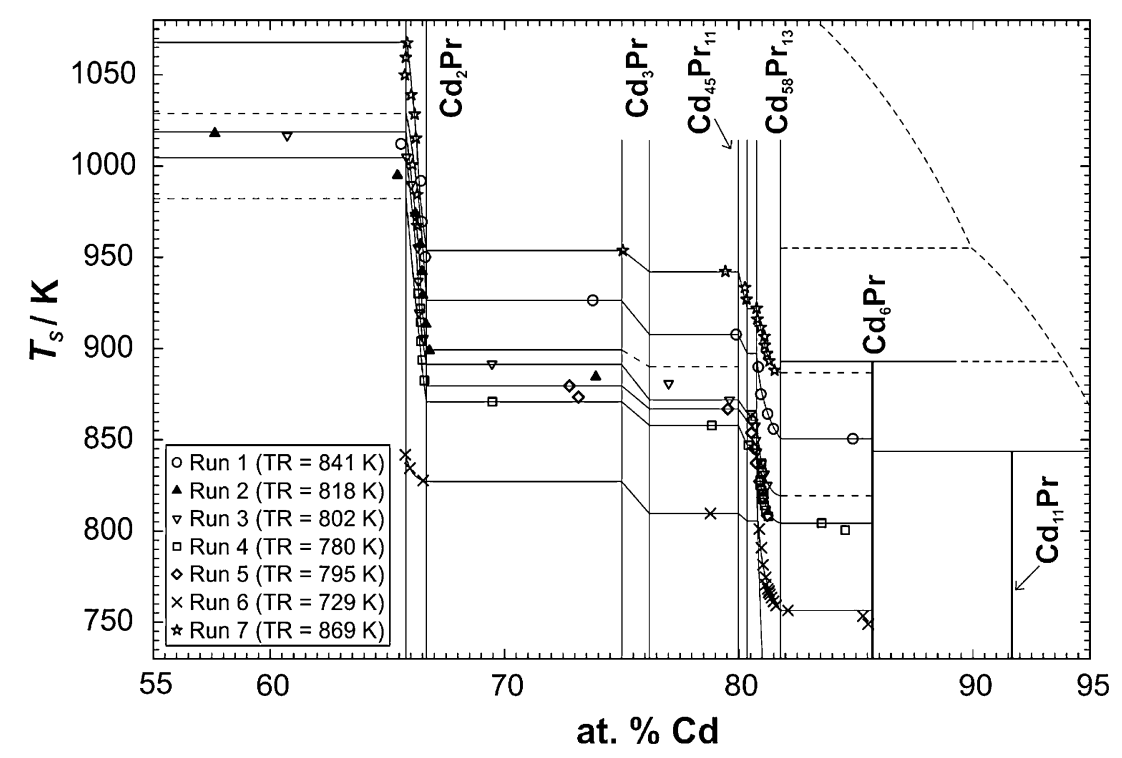

Fig. 4-Sample temperature against sample composition superimposed on the partial Cd-Pr phase diagram.

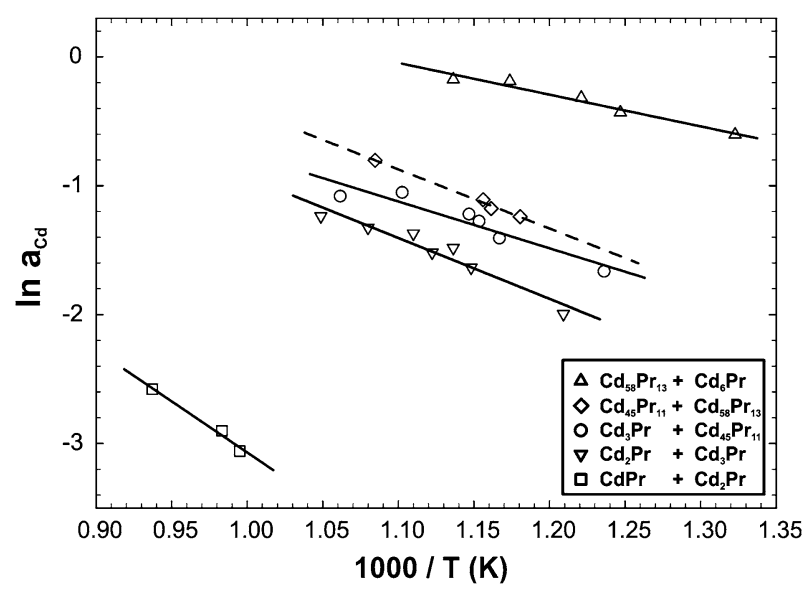

Fig. 5-Natural logarithm of the Cd activity against reciprocal temperature for different two-phase fields.

$$
\frac{\partial \ln a_{\mathrm{Cd}}}{\partial(1 / T)}=\frac{\Delta \bar{H}_{\mathrm{Cd}}}{R}
$$

where the temperature is in $\mathrm{K}, R$ is the gas constant in $\mathrm{J}(\mathrm{mol} \mathrm{K})^{-1}$, and the partial enthalpy of $\mathrm{Cd}$ is in $\mathrm{J} \operatorname{mol}(\mathrm{Cd})^{-1}$. For this approach, straight phase boundaries, i.e., no variation of solid solubilities with temperature, of the different compounds were assumed. Although it is obvious that the solubilities will be temperature dependent in the examined temperature range, this is still a useful approach to obtain information on the corresponding partial enthalpy values in a certain temperature range ( $c f$. Table I). On comparing the slopes in Figure 5, an exothermic behavior is observed in the corresponding composition ranging from 50 to 86 at. pct $\mathrm{Cd}$. One can see that, with decreasing $\mathrm{Cd}$ concentration, the partial molar enthalpy of mixing of $\mathrm{Cd}$ becomes increasingly negative. $\Delta \bar{H}_{\mathrm{Cd}}-$ values for $\mathrm{Cd}_{45} \operatorname{Pr}_{11}+\mathrm{Cd}_{58} \operatorname{Pr}_{13}$ are probably less accu- rate because of the very narrow two-phase field and because of some variation of the solubility of $\mathrm{Cd}_{58} \operatorname{Pr}_{13}$ with temperature, as determined by SEM and included in Figure 4. Therefore, the corresponding straight line in Figure 5 is shown as a dashed line.

\section{Partial Enthalpy of Mixing of $\mathrm{Cd}$ in Single-Phase Fields}

Partial enthalpies of mixing of $\mathrm{Cd}$ were determined in the homogeneity ranges of $\mathrm{Cd}_{2} \mathrm{Pr}$ and $\mathrm{Cd}_{58} \mathrm{Pr}_{13}$ in the same manner as described above. For instance, for the evaluation of $\mathrm{Cd}_{58} \mathrm{Pr}_{13}$ sample temperatures for selected compositions were obtained along the entire homogeneity range by interpolation from the equilibrium curves in Figure 4. From these hypothetical samples, $\mathrm{Cd}$ activities were calculated according to Eqs. [1] and [3]. Natural logarithms of these activities were plotted as a function of reciprocal temperature in Figure 6. Applying the adapted Gibbs-Helmholtz equation [4], a linear regression was applied for data points of each selected composition in the entire homogeneity range of $\mathrm{Cd}_{58} \operatorname{Pr}_{13}$. It can be seen that there is some noticeable scatter, especially in the central part of the phase. However, it should be kept in mind that the data points are spaced in very narrow composition steps, and that small errors in composition and/or temperature in the equilibrium curves in Figure 4 may lead to a noticeable shift in the $\ln a_{\mathrm{Cd}} v s .1 / T$ data points.

Values of $\Delta \bar{H}_{\mathrm{Cd}}$ were directly calculated from the slopes of the straight lines in Figure 6 and plotted against composition in Figure 7 . As can be seen, the partial enthalpy values vary over an extended range from -24 at the $\mathrm{Cd}$-rich side to $-50 \mathrm{~kJ} \operatorname{mol}(\mathrm{Cd})^{-1}$ at the Pr-rich border of the homogeneity range, pointing again to the considerable stability of this phase.

An analogous evaluation was carried out for the $\mathrm{Cd}_{2} \mathrm{Pr}$ phase. The corresponding values are included in Table I. Although the partial enthalpy values in the two- 


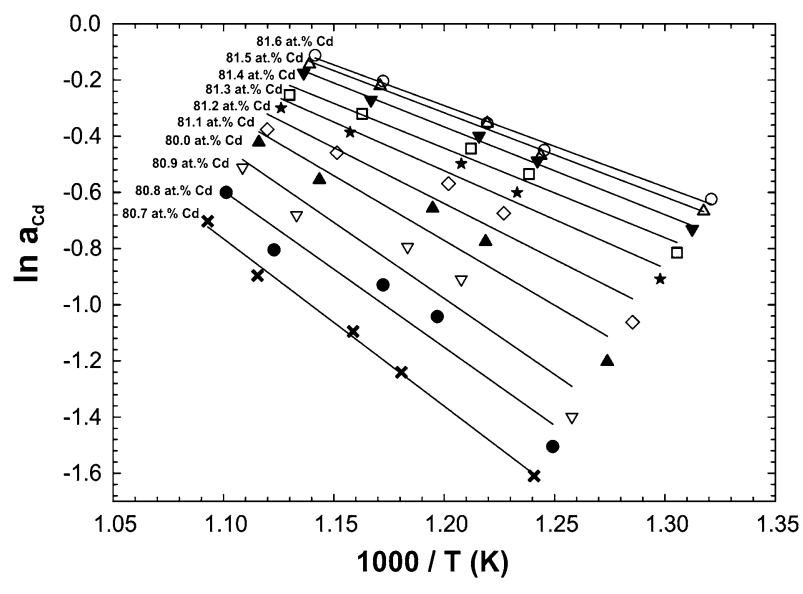

Fig. 6-Natural logarithm of the Cd activity against reciprocal temperature for selected compositions in the $\mathrm{Cd}_{58} \operatorname{Pr}_{13}$ phase.

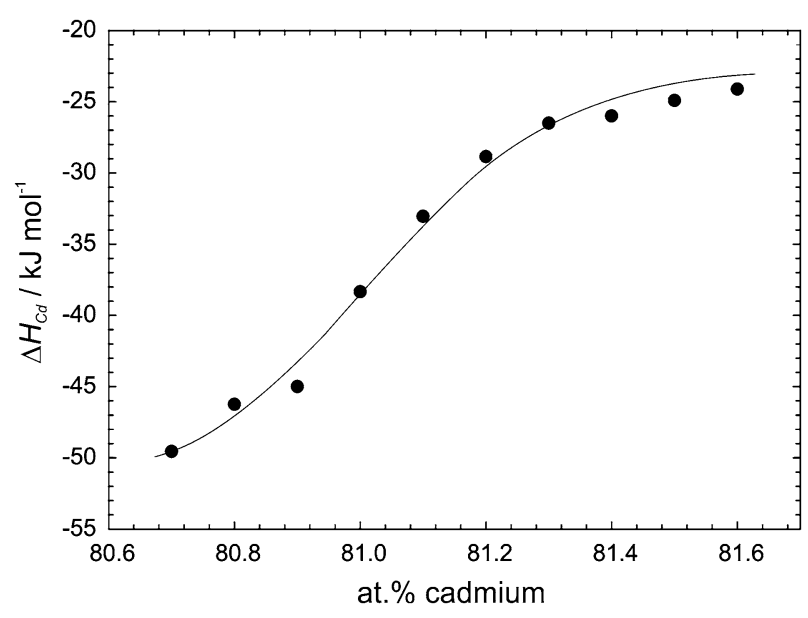

Fig. 7-Partial molar enthalpy of $\mathrm{Cd}$ in the homogeneity range of $\mathrm{Cd}_{58} \operatorname{Pr}_{13}$; standard state: $\mathrm{Cd}(\mathrm{l})$.

phase fields become increasingly more negative with increasing Pr content (see Figure 5), the evaluation for $\mathrm{Cd}_{2} \mathrm{Pr}$ resulted in surprisingly small negative or even slightly positive values for $\Delta \bar{H}_{\mathrm{Cd}}$, which would indicate a somewhat unusual shape of the integral enthalpy of formation curve for this phase. At the moment, the reason for this behavior is not clear; nevertheless, these values were used for the conversion of the $\mathrm{Cd}$ activities to a common temperature of $823 \mathrm{~K}\left(550{ }^{\circ} \mathrm{C}\right.$ ) (see below), resulting in activity values consistent with those of the neighboring phases.

Partial enthalpy values for $\mathrm{Cd}_{45} \mathrm{Pr}_{11}$ could not be derived in the described way, but were estimated from the neighboring two-phase fields assuming a linear behavior with composition between the values of the two-phase fields. The same procedure was employed for the $\mathrm{Cd}_{3} \operatorname{Pr}$ phase where no samples were available from the experiment ( $c f$. Table I).

\section{Thermodynamic Activity of Cd at $823 \mathrm{~K}\left(550^{\circ} \mathrm{C}\right)$}

To derive thermodynamic activities of $\mathrm{Cd}$ for the respective single- and two-phase fields, an integrated

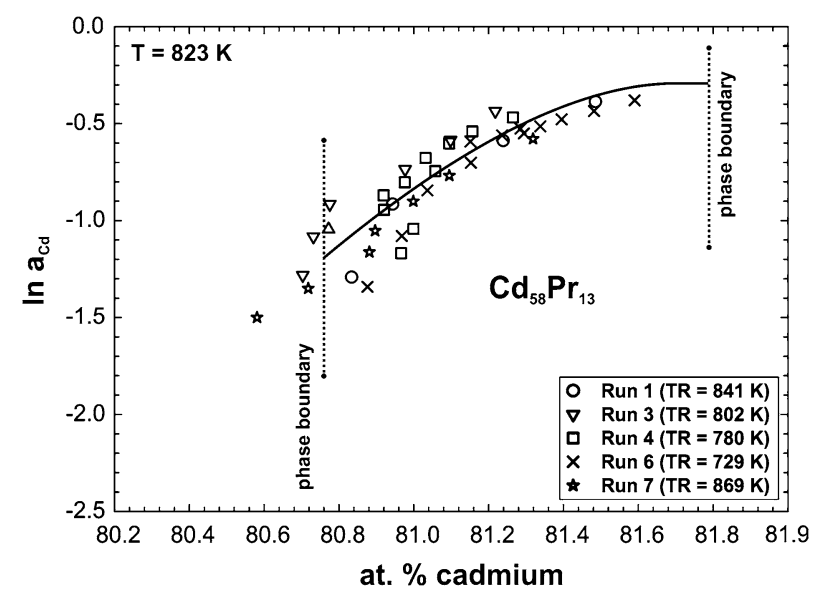

Fig. 8-Natural logarithm of the Cd activity for $\mathrm{Cd}_{58} \operatorname{Pr}_{13}$ at $823 \mathrm{~K}$ $\left(550^{\circ} \mathrm{C}\right)$; standard state: $\mathrm{Cd}(\mathrm{l})$. The symbols are same as in Fig. 4.

form of the adapted Gibbs-Helmholtz equation was applied:

$\ln a_{\mathrm{Cd}}\left[823 \mathrm{~K}\left(550^{\circ} \mathrm{C}\right)\right]-\ln a_{\mathrm{Cd}}\left(T_{\mathrm{S}}\right)=\frac{\Delta \bar{H}_{\mathrm{Cd}}}{R} \cdot\left(\frac{1}{823}-\frac{1}{T_{\mathrm{S}}}\right)$

with the temperature in $\mathrm{K}$ and the partial enthalpy of cadmium in $\mathrm{J}$ mol$(\mathrm{Cd})^{-1}$. Accordingly, the activity values of all samples, at the respective sample temperatures $T_{\mathrm{S}}$, were converted to an average temperature of $823 \mathrm{~K}\left(550{ }^{\circ} \mathrm{C}\right)$ (see Table I). The required enthalpy values in the respective single- and two-phase fields $\left(\mathrm{Cd}_{2} \mathrm{Pr}, \quad \mathrm{Cd}_{2} \mathrm{Pr}+\mathrm{Cd}_{3} \mathrm{Pr}, \mathrm{Cd}_{3} \mathrm{Pr}, \mathrm{Cd}_{3} \mathrm{Pr}+\mathrm{Cd}_{45} \mathrm{Pr}_{11}\right.$, $\mathrm{Cd}_{45} \operatorname{Pr}_{11}, \mathrm{Cd}_{45} \operatorname{Pr}_{11}+\mathrm{Cd}_{58} \operatorname{Pr}_{13}, \mathrm{Cd}_{58} \operatorname{Pr}_{13}, \mathrm{Cd}_{58} \operatorname{Pr}_{13}+$ $\mathrm{Cd}_{6} \mathrm{Pr}$ ) were obtained from the current results.

As an example, $\ln a_{\mathrm{Cd}}$ for $\mathrm{Cd}_{58} \operatorname{Pr}_{13}$ is shown in Figure 8 as a function of composition at $823 \mathrm{~K}$ $\left(550{ }^{\circ} \mathrm{C}\right)$. The corresponding phase boundaries at this temperature were taken from Table II, as obtained from SEM measurements. The data points were fitted with a polynomial function, leading to the best compatibility with the activity values in the adjacent two-phase fields.

As mentioned previously, numerous samples were located in the homogeneity range of $\mathrm{Cd}_{2} \mathrm{Pr}$, making this phase useful to perform the same evaluation as was done for $\mathrm{Cd}_{58} \operatorname{Pr}_{13}$. The homogeneity range was estimated to be between 65.8 and 66.6 at. pct $\mathrm{Cd}$ at $823 \mathrm{~K}\left(550{ }^{\circ} \mathrm{C}\right)$. The corresponding activity values of samples located in $\mathrm{Cd}_{2} \mathrm{Pr}$ were converted to $823 \mathrm{~K}\left(550{ }^{\circ} \mathrm{C}\right)$, using an empirical fit of the respective enthalpy values within this composition range.

Thermodynamic activity values at $823 \mathrm{~K}\left(550{ }^{\circ} \mathrm{C}\right)$ for the phases $\mathrm{Cd}_{3} \operatorname{Pr}$ and $\mathrm{Cd}_{45} \operatorname{Pr}_{11}$ which are given in Table I had to be converted from their corresponding sample temperatures with enthalpy values estimated by a linear interpolation between the values in the neighboring two-phase fields, as discussed above.

Activity values in the two-phase fields $\mathrm{Cd}_{11} \mathrm{Pr}+\mathrm{L}$ and $\mathrm{Cd}_{6} \operatorname{Pr}+\mathrm{Cd}_{11} \operatorname{Pr}$ could not be obtained from the current results but were taken from Johnson and Yonco $^{[13]}$ and Castrillejo et al.,${ }^{[14]}$ respectively, based on their experimental values of the Pr activity and their 


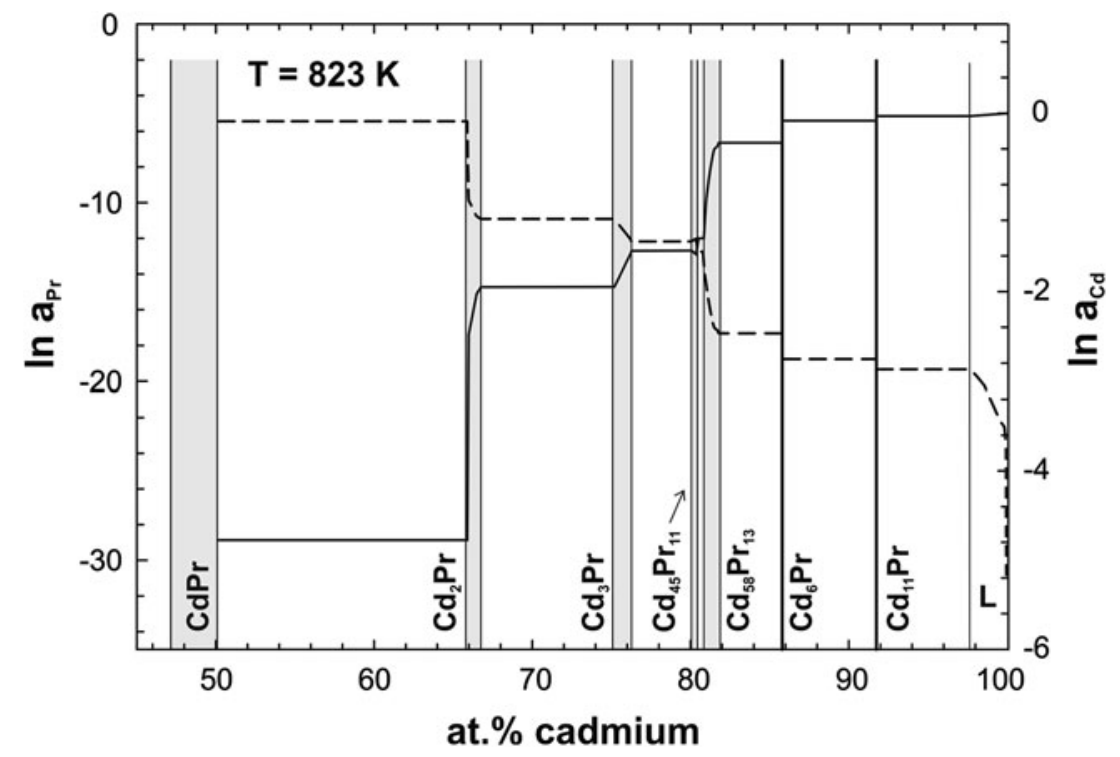

Fig. 9-Natural logarithm of $\mathrm{Cd}$ and $\operatorname{Pr}$ activities against composition at $823 \mathrm{~K}\left(550^{\circ} \mathrm{C}\right)$; standard states: $\alpha$ - $\operatorname{Pr}(\mathrm{s})$ and $\mathrm{Cd}(1)$.

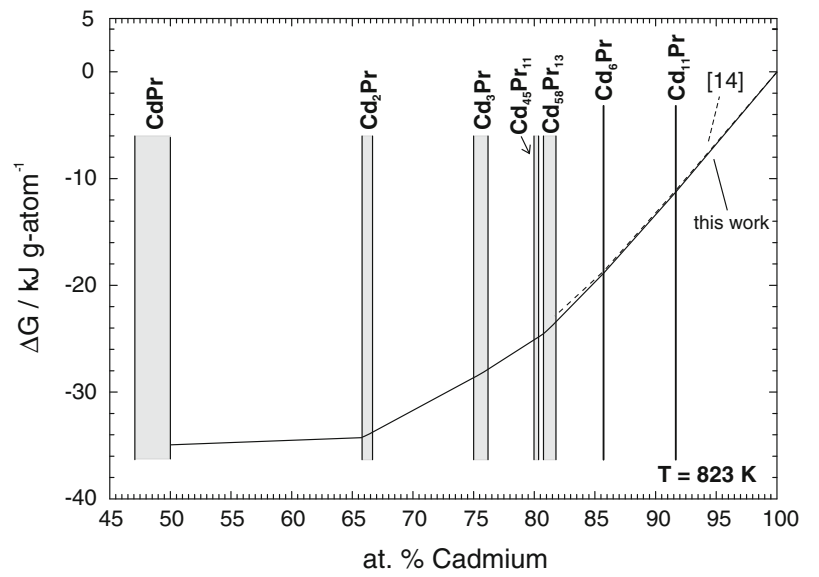

Fig. 10-Integral Gibbs energy of formation against composition at $823 \mathrm{~K}\left(550^{\circ} \mathrm{C}\right)$; standard states: $\alpha-\operatorname{Pr}(\mathrm{s})$ and $\mathrm{Cd}(\mathrm{l})$. Dashed lines refer to Castrillejo et al. ${ }^{[14]}$

integral Gibbs energies of formation of the compounds $\mathrm{Cd}_{6} \operatorname{Pr}$ and $\mathrm{Cd}_{11} \operatorname{Pr}$. They were used for the current Gibbs-Duhem integration as described below. According to Castrillejo et al., ${ }^{[14]}$ a value of $\ln a_{\mathrm{Cd}}=-0.574$ could be calculated for the two-phase field $\mathrm{Cd}_{6} \mathrm{Pr}+$ $\mathrm{Cd}_{58} \operatorname{Pr}_{13}$, which compares reasonably well with the value of about -0.33 obtained in the current study.

\section{E. Integral Gibbs Energy}

As outlined above, Johnson and Yonco ${ }^{[13]}$ determined thermodynamic activities of $\mathrm{Pr}$ in the liquid phase between 635 and $809 \mathrm{~K}\left(362{ }^{\circ} \mathrm{C}\right.$ and $\left.536{ }^{\circ} \mathrm{C}\right)$ by means of emf measurements. They calculated partial excess Gibbs energies of Pr, based on solubility data of $\operatorname{Pr}$ in liquid $\mathrm{Cd}$ from an earlier examination of the $\mathrm{Cd}$-rich part of the phase diagram by Johnson et al. ${ }^{[12]}$ An overall fit of the complete data was given, which was used to calculate an activity coefficient of $\operatorname{Pr}$ at $823 \mathrm{~K}$ $\left(550{ }^{\circ} \mathrm{C}\right)$ for the current study:

$$
\Delta \bar{G}_{\mathrm{Pr}}^{\mathrm{xs}}=x_{\mathrm{Cd}}^{2}\left(a_{1}+a_{2} T+B x_{\mathrm{Pr}}\right)
$$

with the temperature in $\mathrm{K}$ and the partial excess Gibbs energy of $\operatorname{Pr}$ in $\mathrm{J}$ mol$(\mathrm{Pr})^{-1}$. The respective coefficients, obtained from the emf measurements, were listed as follows: $a_{1}=-182.25 \mathrm{~J} \mathrm{~mol}^{-1}, a_{2}=87.50 \mathrm{~J}(\mathrm{~mol} \mathrm{~K})^{-1}$, and $B=-86.67 \times 10^{3} \mathrm{~J} \mathrm{~mol}^{-1}$. From the calculated excess partial Gibbs energy of $\operatorname{Pr}$ at $823 \mathrm{~K}\left(550^{\circ} \mathrm{C}\right)$, a value for the thermodynamic activity of $\operatorname{Pr}$ could be obtained for the two-phase field $\mathrm{Cd}_{11} \mathrm{Pr}+\mathrm{L}$, namely, $\ln a_{\mathrm{Pr}}=-19.34$, which agrees perfectly with the activity value of Castrillejo et al. ${ }^{[14]}$ given as -19.55 . The activity value $\ln a_{\operatorname{Pr}}=-19.34$, calculated from the empirical function of Johnson and Yonco, served as an integration constant for Gibbs-Duhem integration performed in the current study. The corresponding activity values of $\mathrm{Cd}$ and $\mathrm{Pr}$ in the composition ranging from 50 to 100 at. pet $\mathrm{Cd}$ are plotted as natural logarithms in Figure 9.

Again, the figure indicates that the intermetallic compounds $\mathrm{Cd}_{2} \operatorname{Pr}$ and $\mathrm{Cd}_{58} \operatorname{Pr}_{13}$ have to be very stable, because of the marked difference of the activities between their adjacent two-phase fields. Incidentally, it was observed in the Cd-La system ${ }^{[18]}$ that the two phases $\mathrm{Cd}_{2} \mathrm{La}$ and $\mathrm{Cd}_{58} \mathrm{La}_{13}$ with the same crystal structures show congruent melting points, and a similar marked activity change was also observed for the compound $\mathrm{Cd}_{58} \mathrm{La}_{13}$ by Richter et al. ${ }^{[19]}$ Thus, the results for the $\mathrm{Cd}-\mathrm{Pr}$ system are taken as a strong indication that $\mathrm{Cd}_{2} \operatorname{Pr}$ and $\mathrm{Cd}_{58} \operatorname{Pr}_{13}$ might also exhibit congruent melting behavior.

From the activity data for $\mathrm{Cd}$ and $\mathrm{Pr}$, integral Gibbs energies were calculated for the respective composition ranges at $823 \mathrm{~K}\left(550{ }^{\circ} \mathrm{C}\right)$ and are plotted in Figure 10, referred to $\operatorname{Cd}(1)$ and $\alpha$-Pr(s) as standard states. Gibbs energies of formation at the exact stoichiometric compositions of the phases $\mathrm{Cd}_{6} \operatorname{Pr}, \mathrm{Cd}_{58} \operatorname{Pr}_{13}, \mathrm{Cd}_{45} \operatorname{Pr}_{11}$, 
$\mathrm{Cd}_{3} \mathrm{Pr}$, and $\mathrm{Cd}_{2} \mathrm{Pr}$ were obtained to be about -18.8 , $-23.5,-24.8,-28.7$, and $-33.8 \mathrm{~kJ} \mathrm{~g}$-atom $^{-1}$ at $823 \mathrm{~K}$ $\left(550{ }^{\circ} \mathrm{C}\right)$, respectively. Comparing with previous data of Castrillejo et $a l .^{[14]}$ (see Figure 10) who presented a Gibbs energy of formation for $\mathrm{Cd}_{58} \operatorname{Pr}_{13}$ as $-23.7 \mathrm{~kJ}$ g-atom $^{-1}$ [at $823 \mathrm{~K}\left(550{ }^{\circ} \mathrm{C}\right)$ ], the agreement is considered very good. In a thermodynamic assessment, Kurata and Sakamura ${ }^{[20]}$ listed optimized parameters for the Gibbs energy of formation of $\mathrm{Cd}_{6} \mathrm{Pr}$. Using their parameters, a value of about $-18.7 \mathrm{~kJ} \mathrm{~g}$-atom $^{-1}$ was calculated for $823 \mathrm{~K}\left(550{ }^{\circ} \mathrm{C}\right)$, referred to $\mathrm{Cd}(1)$ and $\alpha-\operatorname{Pr}(\mathrm{s})$. This similarity of good agreement confirms the validity of the assumptions made for the Gibbs-Duhem integration performed in the current study.

\section{SUMMARY}

Seven successful vapor pressure runs were carried out, using a (non-)isothermal isopiestic method. Liquid Cd served as the volatile component and was equilibrated with pure $\mathrm{Pr}$ samples between $749 \mathrm{~K}$ and $1067 \mathrm{~K}\left(476{ }^{\circ} \mathrm{C}\right.$ and $794^{\circ} \mathrm{C}$ ). From the primary results, equilibrium curves were drawn and thermodynamic activity values of $\mathrm{Cd}$ were derived in the composition ranging from 50 to 85 at. pct $\mathrm{Cd}$. An adapted Gibbs-Helmholtz equation was applied to convert thermodynamic activity values of $\mathrm{Cd}$ to an average sample temperature of $823 \mathrm{~K}\left(550{ }^{\circ} \mathrm{C}\right)$. With additional information from the literature concerning a value for the activity of Pr in the two-phase field $\mathrm{Cd}_{11} \mathrm{Pr}+\mathrm{L}$, a Gibbs-Duhem integration was performed in the current study. Activity values of $\mathrm{Cd}$ and $\mathrm{Pr}$ are thus presented in the composition ranging from 50 to 100 at. pct $\mathrm{Cd}$. Moreover, integral Gibbs energies could be derived and are shown for the respective composition ranges. The agreement of Gibbs energies of formation with the available literature data is good.

\section{ACKNOWLEDGMENTS}

The authors wish to thank the Austrian Science Fund (FWF) for financial support of this study under
Project No. P 23270-N19, and the Scientific-Technical Cooperation between Austria and India (Project No. IN 05/2011). Moreover, the Faculty of Physics of the University of Vienna and especially Dr. Stephan Puchegger are thanked for the help extended to the authors during the SEM measurements.

\section{REFERENCES}

1. D. Olander: J. Nucl. Mater., 2009, vol. 389, pp. 1-22.

2. I. Johnson: J. Nucl. Mater., 1988, vol. 154, pp. 169-80.

3. J.P. Ackerman: Ind. Eng. Chem. Res., 1991, vol. 30, pp. 141-45.

4. J.J. Laidler, J.E. Battles, W.E. Miller, J.P. Ackerman, and E.L. Carls: Prog. Nucl. Energy, 1997, vol. 31, pp. 131-40.

5. H. Yamana, N. Wakayama, N. Souda, and H. Moriyama: J. Nucl. Mater., 2000, vol. 278, pp. 37-47.

6. H. Moriyama, S. Seshimo, K. Moritani, Y. Ito, and T. Mitsugashira: J. Alloys Compd., 1994, vols. 213-214, pp. 354-59.

7. H. Moriyama, H. Yamana, S. Nishikawa, S. Shibata, N. Wakayama, Y. Miyashita, K. Moritani, and T. Mitsugashira: J. Alloys Compd., 1998, vols. 271-273, pp. 587-91.

8. O. Conocar, N. Douyere, J.-P. Glatz, J. Lacquement, R. Malmbeck, and J. Serp: Nucl. Sci. Eng., 2006, vol. 153, pp. 25361.

9. M. Kurata, Y. Sakamura, T. Hijikata, and K. Kinoshita: J. Nucl. Mater., 1995, vol. 227, pp. 110-21.

10. B. Skołyszewska-Kühberger, T.L. Reichmann, R. Ganesan, and H. Ipser: CALPHAD, 2013. DOI:10.2016/j.calphad.2013. 07.005 .

11. K.A. Gschneidner, Jr. and F.W. Calderwood: Bull. Alloy Phase Diagr., 1988, vol. 9, pp. 130-32.

12. I. Johnson, K.E. Anderson, and R.A. Blomquist: Trans. ASM, 1966, vol. 59, pp. 352-55.

13. I. Johnson and R.M. Yonco: Metall. Trans., 1970, vol. 1, pp. 90510 .

14. Y. Castrillejo, M.R. Bermejo, P.D. Arocas, A.M. Martínez, and E. Barrado: J. Electroanal. Chem., 2005, vol. 579, pp. 343-58.

15. H. Ipser, R. Krachler and K.L. Komarek: in Thermochemistry of Alloys, H. Brodowsky and H.-J. Schaller, eds., Kluwer Academic Publishers, Dordrecht, 1989, pp. 293-306.

16. M. Binnewies and E. Milke: Thermochemical Data of Elements and Compounds, WileyVCH, Weinheim, 1999, p. 295.

17. K.A. Gschneidner, Jr. and F.W. Calderwood: Bull. Alloy Phase Diagr., 1988, vol. 9, pp. 130-31.

18. K.A. Gschneidner, Jr. and F.W. Calderwood: Bull. Alloy Phase Diagr., 1988, vol. 9, pp. 32-34.

19. K. Richter, S. Besana, G. Borzone, and H. Ipser: J. Alloys Compd., 2004, vol. 365, pp. 181-87.

20. M. Kurata and Y. Sakamura: J. Phase Equilib., 2001, vol. 22, pp. 232-40. 